\title{
Applying an Extended Fuzzy Parametric Approach to the Problem of Water Allocations
}

\author{
T. Y. Xu' ${ }^{1}$ and X. S. Qin ${ }^{1,2}$ \\ ${ }^{1}$ School of Civil \& Environmental Engineering, Nanyang Technological University, Blk N1-01c-82, \\ 50 Nanyang Avenue, Singapore 639798 \\ ${ }^{2}$ Earth Observatory of Singapore (EOS), Nanyang Technological University, Singapore 639798 \\ Correspondence should be addressed to X. S. Qin; xsqin@ntu.edu.sg
}

Received 22 November 2012; Accepted 22 January 2013

Academic Editor: Songlin Nie

Copyright (C) 2013 T. Y. Xu and X. S. Qin. This is an open access article distributed under the Creative Commons Attribution License, which permits unrestricted use, distribution, and reproduction in any medium, provided the original work is properly cited.

\begin{abstract}
An extended fuzzy parametric programming (EFPP) model was proposed for supporting water resources allocation problems under uncertainty. EFPP deals with flexible constraints (i.e., fuzzy relationships) by allowing violation of constraints at certain satisfaction degrees (i.e., $\alpha$ levels) and employs fuzzy ranking method to handle trapezoidal-shaped fuzzy coefficients. The objective function is defuzzified by using $\beta$ cuts and weighting factors. The applicability of EFPP was demonstrated by a numerical example and a water resources allocation case. A series of decision alternatives at various satisfaction degrees were obtained. Generally, the higher the $\alpha$ level, the lower the system benefit. In comparison, the $\beta$ level in the objective function posed less sensitive impacts on both objective function and model solutions. The reliability of EFPP was tested by comparing its solutions with those from fuzzy chance constrained programming (FCCP). The results indicated that EFPP performed equally well with FCCP in addressing parameter uncertainties, but it demonstrated a wider applicability due to its extended capacity of handling fuzzy relationships in the model constraints.
\end{abstract}

\section{Introduction}

Water resources allocation is an important task for distributing water resources to various users for ensuring healthy socioeconomic development and ecoenvironmental protection. The task is especially critical for areas that are currently suffering from water scarcity problems and facing even greater challenges under future climate change. The conflict among different water users is hardly avoidable, but application of management models, that fully consider the uneven spatial and temporal distributions of water resources, the interactions between water supply and demand, and the regulatory requirement of local authorities, will surely benefit the related allocation and planning processes. In recent years, it has been recognized that the intrinsic uncertainties linking with many system components in water resources allocation could also affect the effectiveness of management strategies that are normally made based on deterministic conditions. These uncertainties could be related to water availability (e.g., fluctuating hydrological condition), water demand (e.g., growing population and changing weather), transportation/storage loss, water prices, and even human judgment (e.g., regulatory policies).

The previous research efforts relied heavily on stochastic, fuzzy and interval techniques in tackling uncertainties [1-6]. Among various alternatives, fuzzy mathematical programming (FMP) was found effective in dealing with uncertainties caused by measurement errors, implicit knowledge, and ambiguous human judgment. The definition of the fuzzy parameters in FMP has less strict data requirement than that of stochastic ones, and the fuzzy parameters contain richer distribution information than interval numbers $[7,8]$. For decades, many types of FMP models were proposed for solving water resources management problems [9-11]. Depending on the way of handling uncertainties, FMP can be categorized into fuzzy flexible (e.g., fuzzy parametric programming) (FF) [12-14], fuzzy possibilistic (FP) (e.g., fuzzy chance constrained programming) [15-17], and fuzzy 
robust (FR) [18, 19] programming models. Maqsood et al. [20] incorporated fuzzy flexible programming into a two-stage stochastic optimization framework, which embedded risk information into the constraints and objective function. Nie et al. [21] advanced a water management model based on fuzzy robust programming approach for water quality problem, where the model could reflect a compromise between system stability and optimality. Xu and Qin [5] proposed a double-sided fuzzy chance-constrained model and applied it to an agricultural water quality management problem. The proposed model could tackle uncertainties expressed as possibilistic distributions in the constraints and allow system violations at predetermined confidence levels.

The above-mentioned fuzzy approaches have specific scopes of applicability, in the sense of handling (i) fuzziness in objective function and/or constraints and (ii) fuzziness of relationship and/or model parameters. FF programming allows flexibility and elasticity to be reflected in the objective function and constraints but is relatively weak in dealing with ambiguous coefficients $[8,22]$. FP programming tackles fuzzy coefficients in objective function and/or constraints, but is less capable of dealing with fuzzy relationships $[8,22]$. FR programming is designed for handling highly uncertain variables (i.e., dual uncertainties) which are expressed as fuzzy boundary intervals $[19,21]$; it is generally not suitable to be used for reflecting vague relationships or objective functions [5]. In water resources allocation problems, uncertainty could exist in many system components and their relationships. To benefit general-purpose applications, it is desired that a sophisticated model that could handle all possible fuzzy conditions mentioned above will be available.

Herrera and Verdegay [23] gave a general introduction of three models of fuzzy parametric linear programming. These models have shown advantages in dealing with fuzzyrelation-based constraints, fuzzy coefficients in system objective, and fuzzy parameters in model constraints. Although the different types of fuzziness were treated individually, the parametric models did show a potential to be coupled together for handling more complicated cases. This further topic was not discussed in the previous studies, and the applicability of such a method in engineering problems has yet to be explored. In addition, the models were developed for triangular-shaped fuzzy sets and were incapable of reflecting more general cases. Thus, an extended fuzzy parametric programming (EFPP) method, which is based on the models proposed by Herrera and Verdegay [23], will be developed in this study. A numerical example and a water resources allocation problem will be used for demonstration.

\section{Methodology}

2.1. Extended Fuzzy Parametric Programming. A fuzzy linear programming (FLP) problem in consideration of fuzziness under a general condition can be written as follows [24]:

$$
\operatorname{Max} z=\sum_{j=1}^{J} \widetilde{c}_{j} x_{j}
$$

subject to

$$
\begin{gathered}
\sum_{j=1}^{J} \widetilde{a}_{i j} x_{j} \leqslant \widetilde{b}_{i}, \quad \forall i, \\
\sum_{j=1}^{J} d_{j k} x_{j} \leq e_{k}, \quad \forall k, \\
x_{j} \geq 0, \quad \forall j,
\end{gathered}
$$

where $\widetilde{c}_{j}, \widetilde{a}_{i j}$, and $\widetilde{b}_{i}$ are fuzzy coefficients (or parameters); $x_{j}$ is deterministic decision variable, $d_{j k}$ and $e_{k}$ are deterministic coefficients; $j$ is the index of decision variable; $J$ is the number of decision variables; $i$ is the index of fuzzy constraint; $k$ is the index of deterministic constraint; the symbols $\leqslant$ denote fuzzy inequality. The fuzzy coefficients for trapezoidal fuzzy sets could be expressed as $\widetilde{c}_{j}=\left(c_{j}^{1}, c_{j}^{2}, c_{j}^{3}, c_{j}^{4}\right), \tilde{a}_{i j}=$ $\left(a_{i j}^{1}, a_{i j}^{2}, a_{i j}^{3}, a_{i j}^{4}\right)$, and $\widetilde{b}_{i}=\left(b_{i}^{1}, b_{i}^{2}, b_{i}^{3}, b_{i}^{4}\right)$, respectively. In this study, we consider the trapezoidal shape a relatively general shape of a fuzzy membership function. It could address the minimum, maximum, and most possible range of an uncertain variable. The triangular-shaped fuzzy membership function is a special case of trapezoidal ones when the most possible range converges to a single point.

Assume the flexibility of the constraints could be represented by fuzzy sets. When the constraints are fully satisfied, the membership degree of the constraints would be 1, when the constraints are totally violated, the membership degree of the constraints would be 0 . Let a fuzzy number $\widetilde{\theta}_{i}$ represent the allowable maximum violation of the constraints, that is, to be determined by decision makers. The membership degree of constraints $\mu_{i}(x)$ would linearly decrease over the interval $\left(\widetilde{b}_{i}, \widetilde{b}_{i}+\widetilde{\theta}_{i}\right)$, and could be expressed as follows [23]:

$$
\mu_{i}(x)= \begin{cases}1, & \text { if } \sum \widetilde{a}_{i j} x_{j} \leq \widetilde{b}_{i}, \\ \frac{\left[\left(\widetilde{b}_{i}+\widetilde{\theta}_{i}\right)-\sum \widetilde{a}_{i j} x_{j}\right]}{\widetilde{\theta}_{i}}, & \text { if } \widetilde{b}_{i} \leq \sum \widetilde{a}_{i j} x_{j} \leq \widetilde{b}_{i}+\widetilde{\theta}_{i}, \\ 0, & \text { if } \sum \widetilde{a}_{i j} x_{j} \geq \widetilde{b}_{i}+\widetilde{\theta}_{i},\end{cases}
$$

where the fuzziness could exist in both the fulfillment of the constraints and the coefficients in constraints. For simplicity, $\sum_{j=1}^{J}(\cdot)$ is represented by $\sum(\cdot)$. To deal with fuzzy relationship, the fuzzy ranking method (FRM), studied by many researchers [25-28], will be employed. FRM has the following definitions [23]:

$$
\begin{gathered}
\operatorname{FR}(\widetilde{A}) \geq \operatorname{FR}(\widetilde{B}) \Longrightarrow \widetilde{A} \geq \widetilde{B}, \\
\operatorname{FR}(\widetilde{A}+\widetilde{B})=\operatorname{FR}(\widetilde{A})+\operatorname{FR}(\widetilde{B}), \\
\operatorname{FR}(r \widetilde{A})=r \cdot \operatorname{FR}(\widetilde{A}),
\end{gathered}
$$

where $\operatorname{FR}(\cdot)$ is defined as a fuzzy ranking function; $\widetilde{A}$ and $\widetilde{B}$ are fuzzy numbers; $r$ is a deterministic coefficient. Examples of fuzzy ranking functions include Chang's index [25] and 
Yager's first, second, and third indexes [26, 27]. In this study, we use Yager's first index to deal with fuzzy coefficients. Therefore, $\mu_{i}(x)$ in (2) can be transformed to [29]:

$$
\begin{aligned}
\mu_{i}(x) & =\frac{\left[\left(\widetilde{b}_{i}+\widetilde{\theta}_{i}\right)-\sum \tilde{a}_{i j} x_{j}\right]}{\widetilde{\theta}_{i}} \Longrightarrow \mu_{i}(x) \\
& =\frac{\operatorname{FR}\left[\left(\widetilde{b}_{i}+\widetilde{\theta}_{i}\right)-\sum \widetilde{a}_{i j} x_{j}\right]}{\operatorname{FR}\left(\widetilde{\theta}_{i}\right)} .
\end{aligned}
$$

To handle fuzzy parameters, a satisfaction degree (i.e., $\alpha$ ) is introduced. If the decision makers prefer a confidence level of constraint satisfaction to be $\alpha$, the membership degree of the constraints $\mu_{i}(x)$ should be higher than $\alpha$, where $\alpha \in[0,1]$. Then the constraint (lb) could be further transformed to the following [29]:

$$
\begin{aligned}
& \frac{\operatorname{FR}\left(\widetilde{b}_{i}+\tilde{\theta}_{i}-\sum \widetilde{a}_{i j} x_{j}\right)}{\operatorname{FR}\left(\widetilde{\theta}_{i}\right)} \\
& \geq \alpha \Longrightarrow\left[\frac{\operatorname{FR}\left(\widetilde{b}_{i}\right)+\operatorname{FR}\left(\widetilde{\theta}_{i}\right)-\operatorname{FR}\left(\sum \widetilde{a}_{i j} x_{j}\right)}{\operatorname{FR}\left(\widetilde{\theta}_{i}\right)}\right] \\
& \geq \alpha \Longrightarrow \operatorname{FR}\left(\sum \tilde{a}_{i j} x_{j}\right) \leq \operatorname{FR}\left(\widetilde{b}_{i}\right)+(1-\alpha) \cdot \operatorname{FR}\left(\widetilde{\theta}_{i}\right) .
\end{aligned}
$$

Consider the fuzziness in the objective function, the membership degree of the objective function $\mu(z)$ could be expressed in the following trapezoidal form:

$$
\mu(z)= \begin{cases}0, & \text { if } z \leq c_{j}^{1} x_{j}, z \geq c_{j}^{4} x_{j}, \\ \frac{z-c_{j}^{1} x_{j}}{c_{j}^{2} x_{j}-c_{j}^{1} x_{j}}, & \text { if } c_{j}^{1} x_{j} \leq z \leq c_{j}^{2} x_{j}, \\ 1, & \text { if } c_{j}^{2} x_{j} \leq z \leq c_{j}^{3} x_{j}, \\ \frac{c_{j}^{4} x_{j}-z}{c_{j}^{4} x_{j}-c_{j}^{3} x_{j}}, & \text { if } c_{j}^{3} x_{j} \leq z \leq c_{j}^{4} x_{j} .\end{cases}
$$

Equation (6) can be converted into crisp sets using $\beta$-cut, where the range under $\beta$-cut represents the aspiration range of the objective function values that the decision makers would accept. The membership degree $\mu(z)$ should be higher than $\beta$, then we can obtain the following relationship:

$$
\mu(z) \geq \beta \Longrightarrow L(\beta) \leq \sum c_{j} x_{j} \leq U(\beta)
$$

where $L(\beta)=(1-\beta) \sum c_{j}^{1} x_{j}+\beta \sum c_{j}^{2} x_{j}$, and $U(\beta)=\beta \sum c_{j}^{3} x_{j}+$ $(1-\beta) \sum c_{j}^{4} x_{j}$.

Then, it turns into a problem with interval-type objective function, which can be written as $[23,30]$ :

$$
\operatorname{Max}\{z \mid z \in[L(\beta), U(\beta)], \beta \in[0,1]\} .
$$

A simple way to handle the interval objective function is to assign a weight vector $\varepsilon$ to both $L(\beta)$ and $U(\beta)$. In this study, we assume an equal importance of $L(\beta)$ and $U(\beta)$ and use their average as the following objective function:

$$
\operatorname{Max} z=\frac{1}{2}[L(\beta)+U(\beta)]
$$

Then, the fuzzy parametric model can be written as follows:

$$
\begin{array}{r}
\operatorname{Max} z=\frac{1}{2}\left[(1-\beta) \cdot\left(\sum_{j=1}^{J} c_{j}^{1} x_{j}+\sum_{j=1}^{J} c_{j}^{4} x_{j}\right)\right. \\
\left.+\beta \cdot\left(\sum_{j=1}^{J} c_{j}^{2} x_{j}+\sum_{j=1}^{J} c_{j}^{3} x_{j}\right)\right],
\end{array}
$$

subject to

$$
\begin{gathered}
\operatorname{FR}\left(\sum_{j=1}^{J} \widetilde{a}_{i j} x_{j}\right) \leq \operatorname{FR}\left(\widetilde{b}_{i}\right)+(1-\alpha) \cdot \operatorname{FR}\left(\widetilde{\theta}_{i}\right), \quad \forall i, \\
\quad \sum_{j=1}^{J} d_{j k} x_{j} \leq e_{k}, \quad \forall k, \\
x_{j} \geq 0, \quad \alpha, \beta \in[0,1],
\end{gathered}
$$

where $\alpha$ reflects the satisfaction degree of constraints. The selection of $\alpha$ depends on the preference of decision makers. The higher the values of $\alpha$, the higher the satisfaction degree of constraints. If decision makers are willing to make a conservative plan, higher values of $\alpha$ should be selected; conversely, if they prefer a higher objective function value (normally this may lead to higher risk of system violation), lower values of $\alpha$ should be chosen. The parameter $\beta$ represents the level of the aspiration range of objective function. The higher the level of $\beta$, the narrower the range of $[L(\beta), U(\beta)]$. To examine the influence of $\beta$ on the objective function, we could assume that $\beta$ has an increment of $\Delta \beta$. Then, the objective function becomes as follows:

$$
\begin{aligned}
\operatorname{Max} z^{\prime}= & \frac{1}{2}(\beta+\Delta \beta) \\
& \cdot\left[\left(\sum c_{j}^{2} x_{j}+\sum c_{j}^{3} x_{j}\right)-\left(\sum c_{j}^{1} x_{j}+\sum c_{j}^{4} x_{j}\right)\right] \\
& +\frac{1}{2}\left(\sum c_{j}^{1} x_{j}+\sum c_{j}^{4} x_{j}\right) .
\end{aligned}
$$

The equation shows that the influence of $\beta$ on the objective function value relies heavily on the distribution of the fuzzy coefficients. The trend is generally linear provided that the decision variables do not have significant variations.

Figure 1 shows the EFPP's general framework. The steps of using EFPP are as follows: (i) identify fuzzy uncertain parameters and obtain the fuzzy membership function of each variable; (ii) determine the maximum violation of constraints that the decision maker would accept; (iii) establish the aspiration level to the objective function and assign a weight vector to objective function (or use average); (iv) establish 


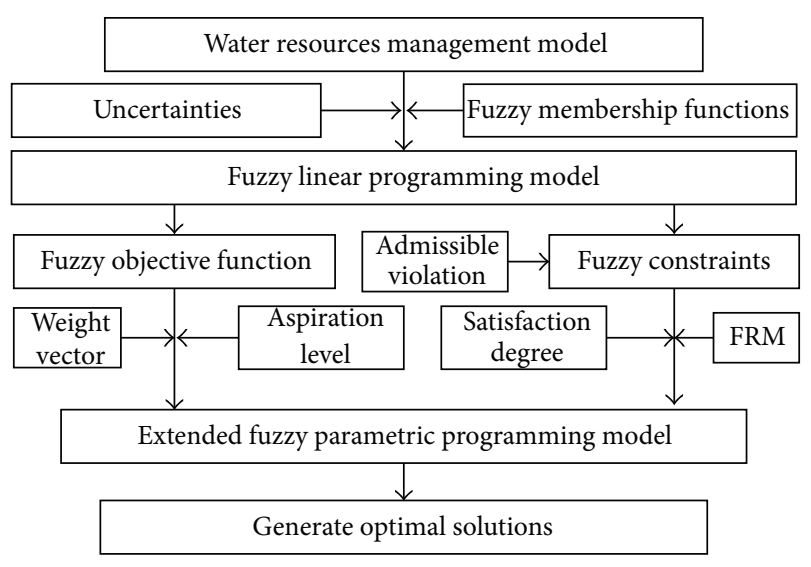

FIGURE 1: General framework of EFPP.

the satisfaction degree to the constraints and transform constraints using FRM; (v) formulate the fuzzy parametric linear programming model and generate the final optimal solutions.

2.2. Numerical Example. Consider the following numerical problem:

$$
\operatorname{Max} f=\widetilde{c}_{1} x_{1}+\widetilde{c}_{2} x_{2}
$$

subjective to

$$
\begin{gathered}
\widetilde{a}_{11} x_{1}-\widetilde{a}_{12} x_{2} \gtrsim \widetilde{b}_{1}, \\
\tilde{a}_{21} x_{1}+\widetilde{a}_{22} x_{2} \leq \widetilde{b}_{2}, \\
x_{j} \geq 0,
\end{gathered}
$$

where $c_{1}=(1,2,3,11), c_{2}=(3,7,8,9), a_{11}=(1,2,2.5,3)$, $a_{12}=(1,2.5,3.5,4), b_{1}=(2,3,5,6), a_{21}=(1.5,3.5,4,6), a_{22}=$ $(3,4,6,7.5), b_{2}=(29,35,36,40)$.

According to (7),

$$
\begin{array}{ll}
l_{1}(\beta)=1+\beta, & u_{1}(\beta)=11-8 \beta, \\
l_{2}(\beta)=3+4 \beta, & u_{2}(\beta)=9-\beta .
\end{array}
$$

The objective function could be written as follows:

$$
\operatorname{Max} f=\left[(1+\beta) x_{1}+(3+4 \beta) x_{2},(11-8 \beta) x_{1}+(9-\beta) x_{2}\right] \text {. }
$$

Assume that $\widetilde{\theta}_{1}=(1,1.5,3,4.5)$ and $\widetilde{\theta}_{2}=(3,5,5.5,7)$ are the admissible violations, and a linear ranking function based on the first index of Yager [27] is used. Then the fuzzy parametric model can be written as follows:

$$
\operatorname{Max} f=\frac{1}{2}\left[(12-7 \beta) x_{1}+(12+3 \beta) x_{2}\right]
$$

subject to

$$
\begin{gathered}
2.125 x_{1}-2.75 x_{2} \geq 4-2.5(1-\alpha), \\
3.75 x_{1}+5.125 x_{2} \leq 35+5.125(1-\alpha), \\
x_{j} \geq 0, \\
\alpha, \beta \in[0,1],
\end{gathered}
$$

where $\alpha$ and $\beta$ are the satisfaction degree of the constraints and the aspiration level of the objective function, respectively. At a $\beta$ level of 0.9 , the results of $\left(f, x_{1}, x_{2}\right)$ obtained from EFPP under $\alpha$ levels of $0.9,0.6$, and 0.3 are $(36.99,5.51,2.89)$, $(39.15,5.53,3.18)$, and $(41.31,5.55,3.47)$, respectively. At an $\alpha$ level of 0.9 , the results of $\left(f, x_{1}, x_{2}\right)$ at $\beta$ levels of $0.9,0.6$, 0.3 are $(36.99,5.51,2.89),(41.48,5.51,2.89)$, and $(46.88,9.47$, $0.315)$, respectively. The results indicate that, as $\alpha$ and $\beta$ levels decrease, the objective function values would both increase. The decision variables would not change until the $\beta$ level drops below 0.3 , which implies that, $\beta$ may only show notable impact on the model solutions when its influence on the objective function reaches a certain threshold.

\section{Application in Water Resources Allocation Problem}

3.1. Case Background and Model Formulation. The same method will be used to a hypothetical water allocation problem [20,31], where two reservoirs are serving as water sources for three users, including municipality, agriculture, and industry. A target water allocation amount to each consuming sector is assigned for each reservoir. Generally, an excessively high water target could lead to water shortage problems when the water availability is low; the corresponding penalties could also be high. Conversely, a too low target may cause waste of water resources during high-flow seasons. Therefore, an optimal water allocation scheme from reservoirs to water users is desired. Thus, the problem under consideration is how to allocate water from various water sources (i.e., reservoirs) to three competing users over three periods so that the overall system benefit can be maximized, while, at the same time, the restrictions of water availability and regulatory requirement should be met.

In such a water resources allocation system, the available water amount is influenced by many factors such as the annual and seasonal variation of rainfall, runoff, and evaporation, as well as groundwater interaction; the values of net benefit and the penalty rely on the market condition and human judgment; the water loss rate is affected by transferring condition (e.g., soil absorption) and infrastructure reliability. Problems of data procurement, survey methods, equipment failure, and human judgment could cause large errors about these parameters. In real-world applications, efforts should be made to ensure an accurate quantification of these uncertainties. In this study, we assume the uncertain parameters be expressed as trapezoidal-shaped fuzzy sets (listed in Tables 1 and 2). 
TABLE 1: Parameters of water demand, reservoir capacity, and water loss rate.

\begin{tabular}{lccc}
\hline & \multicolumn{1}{c}{ Time period } \\
& $t=1$ & \multicolumn{1}{c}{$t=3$} & 11 \\
\hline Municipality & \multicolumn{1}{c}{ Water demand $\left(\times 10^{6} \mathrm{~m}^{3}\right)$} \\
Agriculture & 13 & 10 & 14.5 \\
Industry & 11.5 & 13.5 & 12.5 \\
\hline \multicolumn{4}{c}{ Capacity $\left(\times 10^{6} \mathrm{~m}^{3}\right)$} \\
\hline Reservoir 1 & $(5,5.5,6,6.5)$ & $(5.5,6,6.5,7)$ & $(4,4.5,5,6)$ \\
Reservoir 2 & $(3.5,4.5,5,5.5)$ & $(4,4.5,5.5,6)$ & $(3,3.5,4,5)$ \\
\hline Loss rate & Municipality & Agriculture & Industry \\
\hline Reservoir 1 & $(0.1,0.15,0.18,0.2)$ & $(0.2,0.23,0.25,0.3)$ & $(0.25,0.3,0.35,0.4)$ \\
Reservoir 2 & $(0.05,0.08,0.1,0.15)$ & $(0.1,0.15,0.2,0.25)$ & $(0.15,0.2,0.25,0.3)$ \\
\hline
\end{tabular}

TABLE 2: Parameters of unit net benefit and penalty.

\begin{tabular}{|c|c|c|c|}
\hline & \multicolumn{3}{|c|}{ Time period } \\
\hline & $t=1$ & $t=2$ & $t=3$ \\
\hline \multicolumn{4}{|c|}{ Unit net benefit $\left(\$ / \mathrm{m}^{3}\right)$} \\
\hline \multicolumn{4}{|l|}{ Reservoir 1} \\
\hline Municipality & $(8,10,11,12)$ & $(8.5,11,11.5,12.5)$ & $(10,11,12,14)$ \\
\hline Agriculture & $(3,4.2,4.3,5)$ & $(3.5,4.3,4.5,5.5)$ & $(3.5,4.7,5,6.5)$ \\
\hline Industry & $(6,8,8.3,10)$ & $(7,8,9,10)$ & $(7,8.5,9,11)$ \\
\hline \multicolumn{4}{|l|}{ Reservoir 2} \\
\hline Municipality & $(7,9,9.5,11)$ & $(8,9.5,10.5,12)$ & $(9,10.5,11,13)$ \\
\hline Agriculture & $(2,3.5,3.7,4)$ & $(2,3.5,4,4.5)$ & $(3,4,4.5,5)$ \\
\hline Industry & $(5,7,7.5,9)$ & $(6,7.5,8,9.5)$ & $(6,8,8.5,10)$ \\
\hline \multicolumn{4}{|c|}{ Unit penalty $\left(\$ / \mathrm{m}^{3}\right)$} \\
\hline Municipality & $(15.5,16,18,20)$ & $(16.5,18,20,22)$ & $(18,20,22,24)$ \\
\hline Agriculture & $(5.5,6,7,7.5)$ & $(6,6.5,7,7.5)$ & $(6.5,7,7.5,8)$ \\
\hline Industry & $(11.5,13,14,15)$ & $(12.5,13,15,16.5)$ & $(13.5,15,16,18)$ \\
\hline
\end{tabular}

The model for this water allocation problem can then be formulated as follows:

$$
\text { Maximize } f=\sum_{i=1}^{I} \sum_{j=1}^{J} \sum_{t=1}^{T} N \widetilde{B}_{i j t} \cdot T G_{i j t}-\sum_{i=1}^{I} \sum_{t=1}^{T} \sum_{j=1}^{J} C \widetilde{T}_{i t} \cdot D L_{i j t}
$$

subject to

$$
\begin{gathered}
\sum_{j=1}^{J} T G_{i j t}=T T G_{i t}, \quad \forall i, t, \\
\sum_{i=1}^{I}\left(1+\widetilde{\eta}_{i j}\right) \cdot\left(T G_{i j t}-D L_{i j t}\right) \lesssim I \widetilde{N} F_{j t}, \quad \forall j, t, \\
\sum_{i=1}^{I} \sum_{j=1}^{J} \widetilde{\eta}_{i j} \cdot\left(T G_{i j t}-D L_{i j t}\right) \lesssim T \widetilde{W} L, \quad \forall t, \\
D L_{i j t} \leq T G_{i j t}, \quad \forall i, j, t, \\
T G_{i j t} \geq 0, \quad D L_{i j t} \geq 0, \quad \forall i, j, t,
\end{gathered}
$$

where $i=$ index of water users, and $i=1,2, \ldots, I(I=3) ; j=$ index of reservoirs, and $j=1,2, \ldots, J(J=2)$; $t=$ index of periods, and $t=1,2, \ldots, T,(T=3) ; \eta_{i j}=$ water loss rate from reservoir $j$ to user $i$; $N B_{i j t}=$ unit net benefit of water delivered from reservoir $j$ to user $i$ at period $t\left(\$ / \mathrm{m}^{3}\right) ; T G_{i j t}=$ water allocation target of reservoir $j$ to user $i$ at period $t\left(10^{6} \mathrm{~m}^{3}\right)$; $C T_{i t}=$ unit penalty of not satisfying the target of user $i$ at period $t\left(\$ / \mathrm{m}^{3}\right) ; D L_{i j t}=$ water amount failed to be delivered (i.e., deficit) from reservoir $j$ to user $i$ at period $t\left(10^{6} \mathrm{~m}^{3}\right)$; $T T G_{i t}=$ water allocation target for user $i$ at period $t\left(10^{6} \mathrm{~m}^{3}\right)$; $T W L=$ total allowable water loss $\left(10^{6} \mathrm{~m}^{3}\right) ; I N F_{j t}=$ available water amount for reservoir $j$ at period $t\left(10^{6} \mathrm{~m}^{3}\right)$.

The objective function (16a) is to obtain the maximum system benefit of allocating water from two reservoirs to three consumers. Constraint (16b) means that the total allocation target of reservoirs to each water user should be equal to the demand of each user; constraint (16c) denotes that the total allocated water from each reservoir should be smaller than its available capacity; constraint (16d) means that the total amount of water loss should be lower than the allowable water loss; constraint (16e) denotes that the shortage should 
TABLE 3: Solutions from EFPP.

\begin{tabular}{|c|c|c|c|c|c|c|c|c|c|c|c|c|c|}
\hline & & \multicolumn{3}{|c|}{$\alpha, \beta=0.95$} & \multicolumn{3}{|c|}{$\alpha, \beta=0.9$} & \multicolumn{3}{|c|}{$\alpha, \beta=0.8$} & \multicolumn{3}{|c|}{$\alpha, \beta=0.7$} \\
\hline & & $t=1$ & $t=2$ & $t=3$ & $t=1$ & $t=2$ & $t=3$ & $t=1$ & $t=2$ & $t=3$ & $t=1$ & $t=2$ & $t=3$ \\
\hline \multicolumn{14}{|c|}{ Target from reservoirs to users $\left(\times 10^{6} \mathrm{~m}^{3}\right)$} \\
\hline \multirow{2}{*}{ M } & $\mathrm{R} 1$ & 5.32 & 5.59 & 7.30 & 5.36 & 5.79 & 7.14 & 5.75 & 6.18 & 6.82 & 6.13 & 6.57 & 6.50 \\
\hline & $\mathrm{R} 2$ & 4.38 & 4.41 & 3.70 & 4.34 & 4.21 & 3.86 & 3.95 & 3.82 & 4.17 & 3.57 & 3.43 & 4.50 \\
\hline \multirow{2}{*}{ A } & $\mathrm{R} 1$ & 8.91 & 9.10 & 11.1 & 8.77 & 8.95 & 10.9 & 8.47 & 8.65 & 10.6 & 8.17 & 8.35 & 10.3 \\
\hline & $\mathrm{R} 2$ & 4.09 & 4.40 & 3.45 & 4.23 & 4.55 & 3.60 & 4.53 & 4.85 & 3.89 & 4.83 & 5.15 & 4.19 \\
\hline \multirow{2}{*}{ I } & $\mathrm{R} 1$ & 7.58 & 7.78 & 9.19 & 7.44 & 7.63 & 9.05 & 7.15 & 7.35 & 8.77 & 6.87 & 7.06 & 8.48 \\
\hline & $\mathrm{R} 2$ & 3.92 & 4.22 & 3.31 & 4.06 & 4.37 & 3.45 & 4.35 & 4.65 & 3.73 & 4.63 & 4.94 & 4.02 \\
\hline \multicolumn{14}{|c|}{ Water deficit for users $\left(\times 10^{6} \mathrm{~m}^{3}\right)$} \\
\hline \multirow{2}{*}{ M } & $\mathrm{R} 1$ & 0.15 & 0 & 2.90 & 0 & 0 & 2.54 & 0 & 0 & 1.83 & 0 & 0 & 1.12 \\
\hline & $\mathrm{R} 2$ & 0 & 0 & 0 & 0 & 0 & 0 & 0 & 0 & 0 & 0 & 0 & 0 \\
\hline \multirow{2}{*}{ A } & R1 & 4.12 & 3.89 & 6.96 & 3.79 & 3.57 & 6.63 & 3.13 & 2.91 & 5.97 & 2.47 & 2.25 & 5.31 \\
\hline & R2 & 0 & 0 & 0 & 0 & 0 & 0 & 0 & 0 & 0 & 0 & 0 & 0 \\
\hline \multirow{2}{*}{ I } & R1 & 3.07 & 2.89 & 5.34 & 2.76 & 2.58 & 5.03 & 2.13 & 1.95 & 4.41 & 1.51 & 1.33 & 3.78 \\
\hline & $\mathrm{R} 2$ & 0 & 0 & 0 & 0 & 0 & 0 & 0 & 0 & 0 & 0 & 0 & 0 \\
\hline
\end{tabular}

M: municipality; A: agriculture; I: industry; R1: reservoir 1; R2: reservoir 2.

not exceed a predefined target; constraint (16f) stipulates the nonnegativity of all decision variables. The fuzzy parameters are associated with unit benefits, unit penalties, water loss rates, allowable water losses, and available water amounts. The fuzzy relations in constraints (16c) and (16d) mean that the total allocation amounts and water loss amounts are not strictly restricted by the policy regulations and violations are allowable to a certain degree (i.e., satisfaction degree). Constraints (16c) and (16d) can be transformed to:

$$
\begin{aligned}
& \sum_{i=1}^{I}\left(1+\widetilde{\eta}_{i j}\right) \cdot\left(T G_{i j t}-D L_{i j t}\right) \lesssim I \widetilde{N} F_{j t}+(1-\alpha) \cdot \widetilde{\theta}_{j t}^{\text {inf }} \\
& \sum_{i=1}^{I} \sum_{j=1}^{J} \widetilde{\eta}_{i j} \cdot\left(T G_{i j t}-D L_{i j t}\right) \lesssim T \widetilde{W} L+(1-\alpha) \cdot \widetilde{\theta}^{t w l},
\end{aligned}
$$

where $\widetilde{\theta}_{j t}^{\inf }$ and $\widetilde{\theta}^{t w l}$ are the acceptable tolerances of water availability and total water loss amount, respectively. Constraint (17a) shows that if the total allocated water from reservoir is higher than its total water availability, the satisfaction degree would decrease. It would not be acceptable if the total allocated water amount exceeds the tolerance of water availability (i.e., $I \widetilde{N} F_{j t}+\widetilde{\theta}_{j t}^{\text {inf }}$ ). Constraint (17b) shows a similar treatment for water losses.

3.2. Results. For simplicity of demonstration, we firstly assume the aspiration level of the objective function is identical to the satisfaction degree of constraints. The fuzzy parameters in constraints are dealt with by the first index of Yager [26, 27]. Table 3 lists the solution of the allocated target and shortage under various satisfaction degrees. For reservoir 1, the water target assigned to agriculture is the highest, followed by industry and municipality. For example, at period 1, under satisfaction degree of 0.9 , the target amounts from reservoir 1 to municipality, agriculture, and industry are $5.36,8.77$, and $7.44\left(\times 10^{6} \mathrm{~m}^{3}\right)$, respectively. This is because the demand of agriculture is the highest and the available flow of reservoir 1 is higher than that of reservoir 2; consequently, more water would be allocated to satisfy the demand of agriculture from reservoir 1. Table 3 also shows that, as the satisfaction degree decreases, the target amount from reservoir 1 to municipality would increase. For example, at period 2, as the satisfaction decreases from 0.95 to 0.7 , the target allocation amount from reservoir 1 to municipality would increase from 5.32 to $6.13\left(\times 10^{6} \mathrm{~m}^{3}\right)$. This is due to the fact that a lower satisfaction level corresponds to a higher violation degree; reservoir 1, which has a higher net benefit, would be preferred to supply more water.

The total shortages of municipality, agriculture, and industry over periods 1 to 3 under various satisfaction degrees have been plotted in Figure 2. The total shortage amount of municipality is the lowest and that of agriculture is the highest. It means that the target of municipality would be satisfied first, and that of agriculture would be of least priority. This is due to the highest net benefit the municipality could bring in and also the highest penalty if the target of municipality could not be satisfied. It also shows that as $\alpha$ level decreases, the shortage amount of the three users would decrease. For example, at period 3, under satisfaction degrees of $0.95,0.9,0.8$, and 0.7 , the total water shortage amount of agriculture are $6.96,6.63,5.97$, and $5.31\left(\times 10^{6} \mathrm{~m}^{3}\right)$, respectively. This implies that, under a lower satisfaction degree (i.e., higher risk of constraint violation), the decision makers would be more optimistic about the water availability and prefer to allocate more water and avoid more shortage problem.

Figure 3 shows the total target and water allocation amount from reservoirs 1 and 2. It demonstrates that the total target amount from reservoir 2 is lower than that from reservoir 1; correspondingly, the total allocated amount would be 


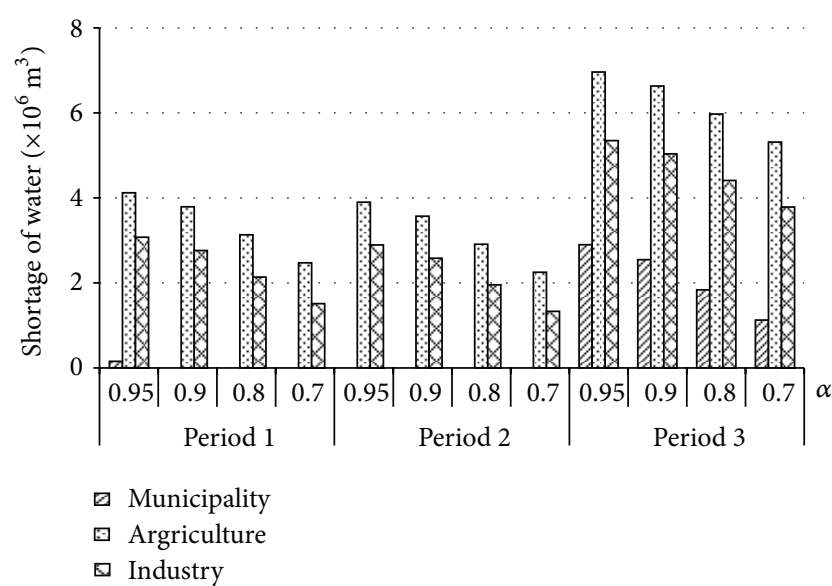

FIGURE 2: Water shortages of municipality, agriculture and industry.

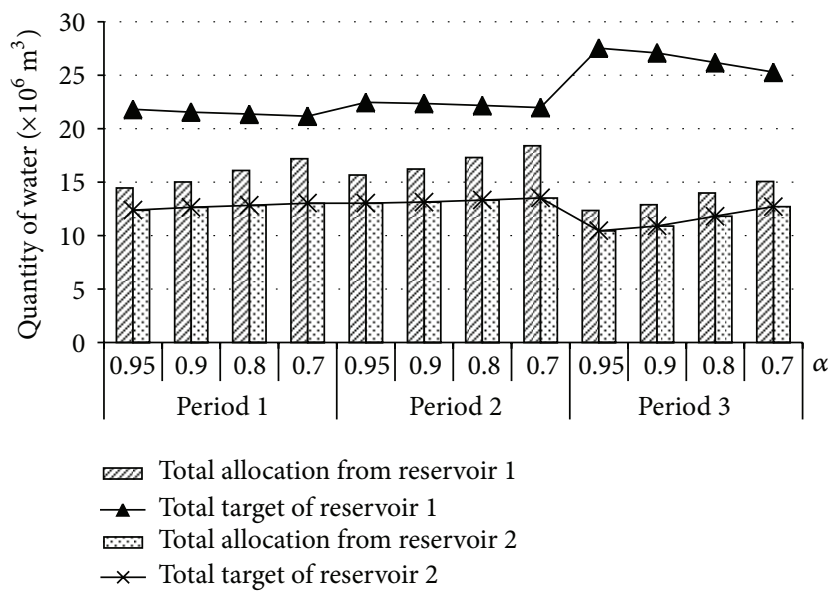

FIgURE 3: Total target and allocation amounts from reservoirs 1 and 2.

lower. For example, at period 1 , under satisfaction degree of 0.9 , the total target from reservoirs 1 and 2 is 21.56 and $12.64\left(\times 10^{6} \mathrm{~m}^{3}\right)$, respectively, and the total allocation is 15.02 and $12.63\left(\times 10^{6} \mathrm{~m}^{3}\right)$, respectively. This is because reservoir 1 has higher net benefit and capacity. It also shows that, as $\alpha$ decreases, the total target from reservoir 1 would decrease and the total allocation amount from reservoir 1 would increase. For example, at period 2, as the satisfaction degree drops from 0.95 to 0.7 , the total target of reservoir 1 would decrease from 22.5 to $22.0\left(\times 10^{6} \mathrm{~m}^{3}\right)$, and the allocation from reservoir 1 would increase from 15.7 to $18.4\left(\times 10^{6} \mathrm{~m}^{3}\right)$. This is because, as the satisfaction degree decreases, the allowable violation of the constraints (i.e., constraints of water available and loss) would increase, and the discrepancy between water target and allocation (i.e., shortage amount) would reduce; this could lead to a higher system benefit.

Figure 4 presents the net benefits of the system at various satisfaction degrees. A higher satisfaction degree has an obvious lower system benefit but a lower risk of system reliability, and vice versa. Generally, the results obtained through EFPP demonstrate that the approach is capable of (i)

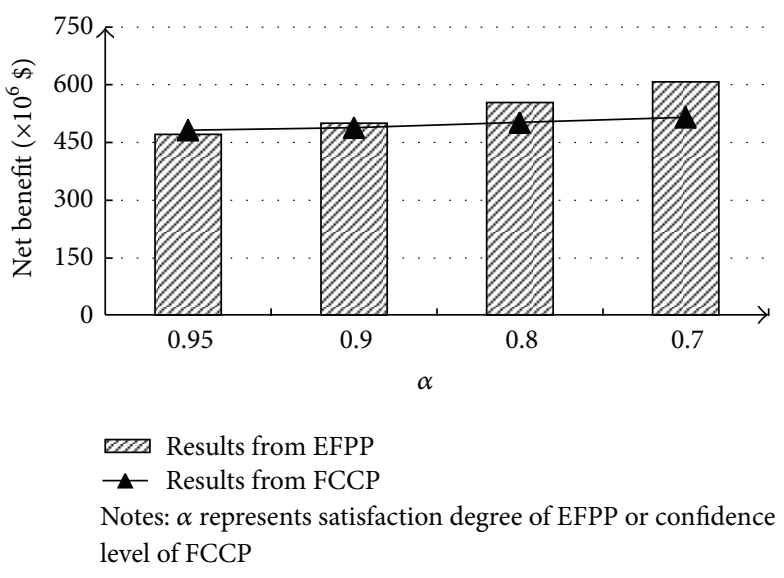

FIGURE 4: System benefits obtained from EFPP and FCCP.

tackling uncertainties in water allocation problems as fuzzy sets; (ii) dealing with fuzzy parameters in both objective function and constraints; (iii) handling fuzzy relationship by allowing violation of the accomplishment of the constraints; (iv) helping decision makers understand the balance between system benefit and reliability.

3.3. Comparison with FCCP. To verify the reliability of the solutions from EFPP, the fuzzy chance constrained programming (FCCP) model, proposed by Liu and Iwamura [32], is applied to solve the same problem after a few modifications. As FCCP could only tackle fuzzy parameters, the treatment of fuzzy relationship is not considered. For simplicity, the fuzzy objective function has been converted to its deterministic version by averaging the upper and lower bounds of the fuzzy coefficients. The fuzzy parameters in the constraints are still expressed as trapezoidal-shaped fuzzy sets. We use confidence levels of $0.95,0.9,0.8$, and 0.7 for the FCCP model; they are deemed equivalent to the satisfaction degrees used in EFPP. The objective-function values obtained from both FCCP and EFPP are shown in Figure 4, and decision variables are shown in Figure 5. At a high satisfaction degree or confidence level (i.e., 0.95), the system benefit obtained from EFPP is slightly lower than that from FCCP. As the satisfaction degree decreases, the system benefits from EFPP would outstrip the results at the same confidence level from FCCP. For example, under satisfaction degrees of 0.9, 0.8, and 0.7, the system benefits from EFPP would be 498.26, 551.79 , and $605.18\left(\times 10^{6} \$\right)$, respectively; those from FCCP at confidence levels of $0.9,0.8$, and 0.7 are $488.90,502.13$, and $515.48\left(\times 10^{6} \$\right)$, respectively.

Figure 5 shows the values of decision variables obtained from FCCP and EFPP. It is obvious that the solutions from both models are fairly close to each other at the same levels of satisfaction or confidence. For instance, at period 3, at a satisfaction degree of 0.7 , the target amounts from reservoir 1 to municipality, agriculture, and industry from EFPP are 6.50, 10.3 , and $8.48\left(\times 10^{6} \mathrm{~m}^{3}\right)$, respectively, and at a confidence level of 0.7 , the target amounts obtained from FCCP are 6.99, 10.7, 


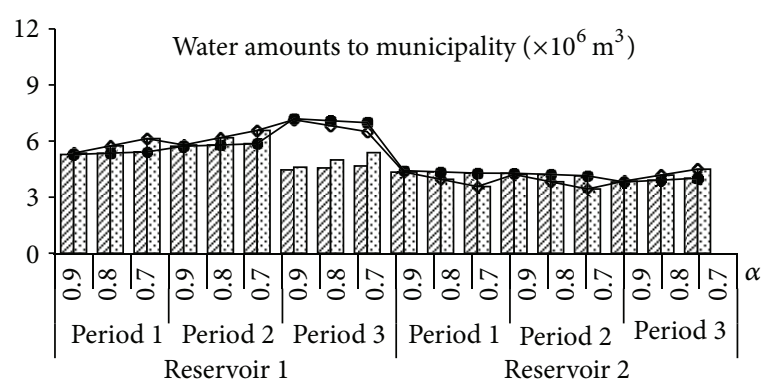

(a)

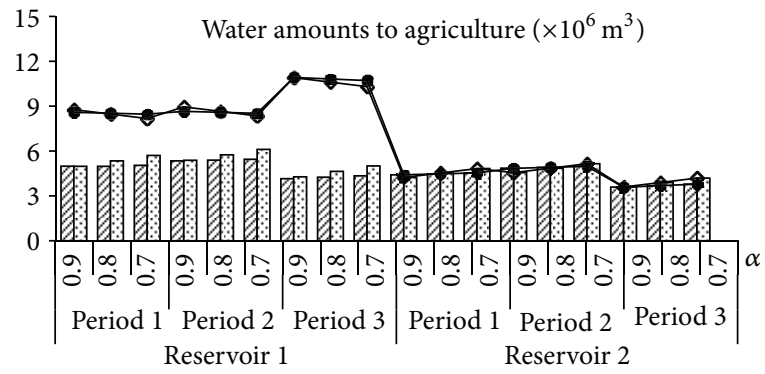

(b)

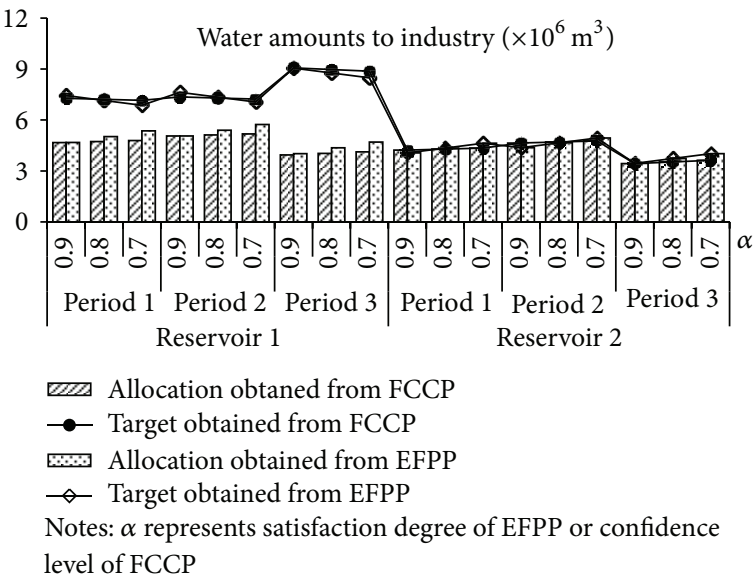

(c)

FIgURE 5: Comparison between FCCP and EFPP.

and $8.87\left(\times 10^{6} \mathrm{~m}^{3}\right)$, respectively. The solutions of allocation from EFPP are somewhat higher than those obtained from FCCP when $\alpha$ level is lower than 0.8; this explains the higher benefits of EFPP shown in Figure 4. From the comparison results, it appears that FCCP and EFPP are both capable of reflecting the balance of system benefit and reliability and could lead to comparable solutions under the same model settings. Obviously, EFPP has a wider applicability due to its extended capacity of handling fuzzy relationships in the model constraints.

3.4. Solutions under Different $\alpha$ and $\beta$ Levels. Figure 6 shows the net benefits under various $\alpha$ and $\beta$ levels. Obviously, the higher the $\alpha$ value, the more reliable the system and the lower the system benefit. For instance, when $\beta=0.9$, the net benefit would increase from 498.26 to $793.68\left(\times 10^{6} \$\right)$ when $\alpha$ level decreases from 0.9 to 0.1 . Figure 6 also shows that the objective function is more sensitive to $\alpha$ than $\beta$. For example, when $\alpha=0.9$, the difference of net benefits between $\beta=0.9$ and 0.1 is $9.23\left(\times 10^{6} \$\right)$, when $\beta=0.9$, the difference of net benefits between $\alpha=0.9$ and 0.1 is $295.42\left(\times 10^{6} \$\right)$. It is also found that the values of decision variables would not vary with the change of $\beta$ for this study case. This is due to the fact that the distributions of fuzzy coefficients in the objective function only cause negligible influence on model solutions; this is consistent with what we have explained in the numerical example section.
3.5. Applicability of EFPP to Other Engineering Management Problems. The proposed method has a potential to be applied to many other engineering management problems, where uncertainties could be described by fuzzy sets. For example, water quality management is also complicated with uncertainties existed among many socioeconomic, environmental, and technical factors, as well as their interactions. The optimization model for water quality management may include environmental constraints, such as the pollutant loading restrictions. The estimation of pollutant loads and maximum allowable discharge amounts involves experience of experts, model estimation errors, and data shortage, and the related parameter uncertainties could be described by fuzzy sets. The decision makers may also accept a certain level of exceedance of environmental constraints, and the unit cost associated with the water quality treatment may also vary with market conditions and subject to uncertainties. EFPP would be most suitable for such type of problems, provided that the model structures and parameters be specifically designed and estimated.

\section{Conclusion}

An extended fuzzy parametric programming (EFPP) approach was developed in this study and applied to a water resource allocation problem. The proposed method could deal with fuzzy parameters with trapezoidal-shaped 


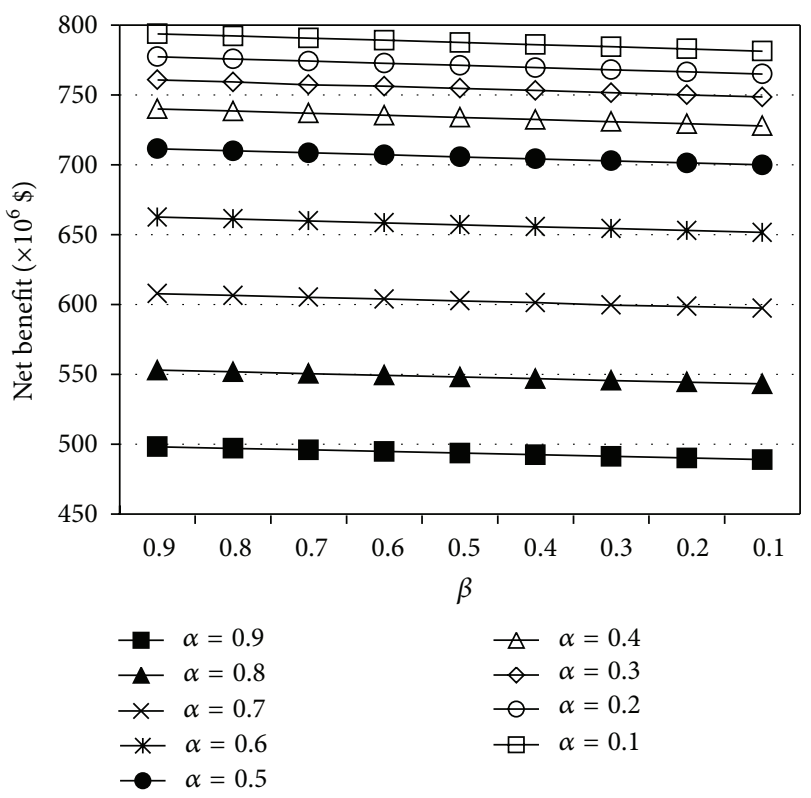

Figure 6: Net benefits under different $\alpha$ and $\beta$ values.

distribution functions in the model, and also fuzzy relationships in the constraints. The results obtained from a water allocation problem showed that EFPP was capable of tackling a wide range of fuzziness in the management model and allowed water managers to analyze the balance of system benefit and risk of failure. Compared with conventional FCCP method, EFPP was flexible in handling the fuzzy relationship and fuzzy parameters in both objective function and constraints and had a more general applicability.

The EFPP method also showed a number of limitations. Firstly, EFPP was restricted to fuzzy variables with triangular- or trapezoidal-shaped membership functions. For more general-shape fuzzy variables, heuristic techniques may be employed [32]. Secondly, the value of using EFPP should also be compared with methods with multiple uncertaintyanalysis techniques such as coupled fuzzy-stochastic theory $[20,31]$ as some particular forms of uncertainty may be better described by other algorithms like interval or stochastic ones. Nevertheless, the proposed method was proved effective in simple water allocation problems, and further applications and verifications in a wider range of engineering fields with more complicated conditions are expected.

\section{Acknowledgment}

This research was supported by Singapore's Ministry of Education (MOM) AcRF Tier 1 Project (M4010973.030).

\section{References}

[1] G. H. Huang, "IPWM: an interval parameter water quality management model," Engineering Optimization, vol. 26, no. 2, pp. 79-103, 1996.
[2] G. H. Huang, "A hybrid inexact-stochastic water management model," European Journal of Operational Research, vol. 107, no. 1, pp. 137-158, 1998.

[3] B. Abolpour and M. Javan, "Optimization model for allocating water in a river basin during a drought," Journal of Irrigation and Drainage Engineering-ASCE, vol. 133, no. 6, pp. 559-572, 2007.

[4] H. W. Chen and N. B. Chang, "Using fuzzy operators to address the complexity in decision making of water resources redistribution in two neighboring river basins," Advances in Water Resources, vol. 33, no. 6, pp. 652-666, 2010.

[5] Y. Xu and X. S. Qin, "Agricultural effluent control under uncertainty: an inexact double-sided fuzzy chance-constrained model," Advances in Water Resources, vol. 33, no. 9, pp. 997-1014, 2010.

[6] M. R. Nikoo, R. Kerachian, and H. Poorsepahy-Samian, "An interval parameter model for cooperative inter-basin water resources allocation considering the water quality issues," Water Resources Management, vol. 26, no. 11, pp. 3329-3343, 2012.

[7] C. Baudrit, D. Guyonnet, and D. Dubois, "Postprocessing the hybrid method for addressing uncertainty in risk assessments," Journal of Environmental Engineering-ASCE, vol. 131, no. 12, pp. 1750-1754, 2005.

[8] M. Inuiguchi and J. Ramík, "Possibilistic linear programming: a brief review of fuzzy mathematical programming and a comparison with stochastic programming in portfolio selection problem," Fuzzy Sets and Systems, vol. 111, no. 1, pp. 3-28, 2000.

[9] R. Slowinski, "A multicriteria fuzzy linear programming method for water supply system development planning," Fuzzy Sets and Systems, vol. 19, no. 3, pp. 217-237, 1986.

[10] J. Kindler, "Rationalizing water requirements with aid of fuzzy allocation model," Journal of Water Resources Planning \& Management-ASCE, vol. 118, no. 3, pp. 308-323, 1992.

[11] C. S. Lee and S. P. Chang, "Interactive fuzzy optimization for an economic and environmental balance in a river system," Water Research, vol. 39, no. 1, pp. 221-231, 2005.

[12] R. M. Faye, S. Sawadogo, and F. Mora-Camino, "Flexible management of water resource systems," Applied Mathematics and Computation, vol. 167, no. 1, pp. 516-527, 2005.

[13] J. Mula, R. Poler, and J. P. Garcia, "MRP with flexible constraints: a fuzzy mathematical programming approach," Fuzzy Sets and Systems, vol. 157, no. 1, pp. 74-97, 2006.

[14] R. R. Tan, "Fuzzy optimization model for source-sink water network synthesis with parametric uncertainties," Industrial and Engineering Chemistry Research, vol. 50, no. 7, pp. 36863694, 2011.

[15] B. Julien, "An extension to possibilistic linear programming," Fuzzy Sets and Systems, vol. 64, no. 2, pp. 195-206, 1994.

[16] H. Tanaka, P. Guo, and H. J. Zimmermann, "Possibility distributions of fuzzy decision variables obtained from possibilistic linear programming problems," Fuzzy Sets and Systems, vol. 113, no. 2, pp. 323-332, 2000.

[17] C. Riverol, M. V. Pilipovik, and C. Carosi, "Assessing the water requirements in refineries using possibilistic programming," Chemical Engineering and Processing, vol. 45, no. 7, pp. 533-537, 2006.

[18] C. Xu and I. C. Goulter, "Optimal design of water distribution networks using fuzzy optimization," Civil Engineering and Environmental Systems, vol. 16, no. 4, pp. 243-266, 1999.

[19] H. Zhu, G. H. Huang, P. Guo, and X. S. Qin, "A fuzzy robust nonlinear programming model for stream water quality management," Water Resources Management, vol. 23, no. 14, pp. 2913-2940, 2009. 
[20] I. Maqsood, G. H. Huang, and J. S. Yeomans, "An intervalparameter fuzzy two-stage stochastic program for water resources management under uncertainty," European Journal of Operational Research, vol. 167, no. 1, pp. 208-225, 2005.

[21] X. H. Nie, G. H. Huang, D. Wang, and H. L. Li, "Robust optimisation for inexact water quality management under uncertainty," Civil Engineering and Environmental Systems, vol. 25, no. 2, pp. 167-184, 2008.

[22] S. A. Torabi and E. Hassini, "An interactive possibilistic programming approach for multiple objective supply chain master planning," Fuzzy Sets and Systems, vol. 159, no. 2, pp. 193-214, 2008.

[23] F. Herrera and J. L. Verdegay, "Three models of fuzzy integer linear programming," European Journal of Operational Research, vol. 83, no. 3, pp. 581-593, 1995.

[24] M. Delgado, J. L. Verdegay, and M. A. Vila, "A general model for fuzzy linear programming," Fuzzy Sets and Systems, vol. 29, no. 1, pp. 21-29, 1989.

[25] W. Chang, "Ranking of fuzzy utilities with triangular membership functions," in Proceedingd of International Conference on Policy Analysis and Information Systems, pp. 263-272, 1981.

[26] R. R. Yager, "Ranking fuzzy subsets over the unit interval," in Proceedings of the International Conference on Decision and Control (CDC '78), pp. 1435-1437, San Diego, Calif, USA, 1978.

[27] R. R. Yager, "A procedure for ordering fuzzy subsets of the unit interval," Information Sciences, vol. 24, no. 2, pp. 143-161, 1981.

[28] R. R. Yager, "Mathematical programming approach to inference with the capability of implementing default rules," International Journal of Man-Machine Studies, vol. 29, no. 6, pp. 685-714, 1988.

[29] J. M. Cadenas and J. L. Verdegay, "Using fuzzy numbers in linear programming," IEEE Transactions on Systems, Man, and Cybernetics, Part B: Cybernetics, vol. 27, no. 6, pp. 1016-1022, 1997.

[30] H. Ishibuchi and H. Tanaka, "Multiobjective programming in optimization of the interval objective function," European Journal of Operational Research, vol. 48, no. 2, pp. 219-225, 1990.

[31] Y. P. Li, G. H. Huang, and S. L. Nie, "An interval-parameter multi-stage stochastic programming model for water resources management under uncertainty," Advances in Water Resources, vol. 29, no. 5, pp. 776-789, 2006.

[32] B. Liu and K. Iwamura, "Chance constrained programming with fuzzy parameters," Fuzzy Sets and Systems, vol. 94, no. 2, pp. 227-237, 1998. 


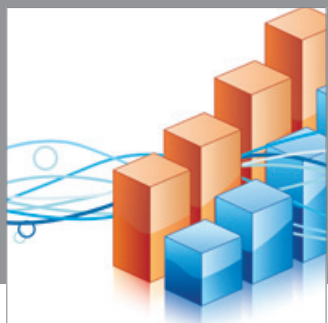

Advances in

Operations Research

mansans

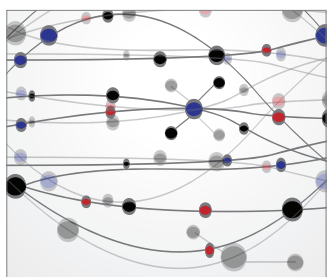

The Scientific World Journal
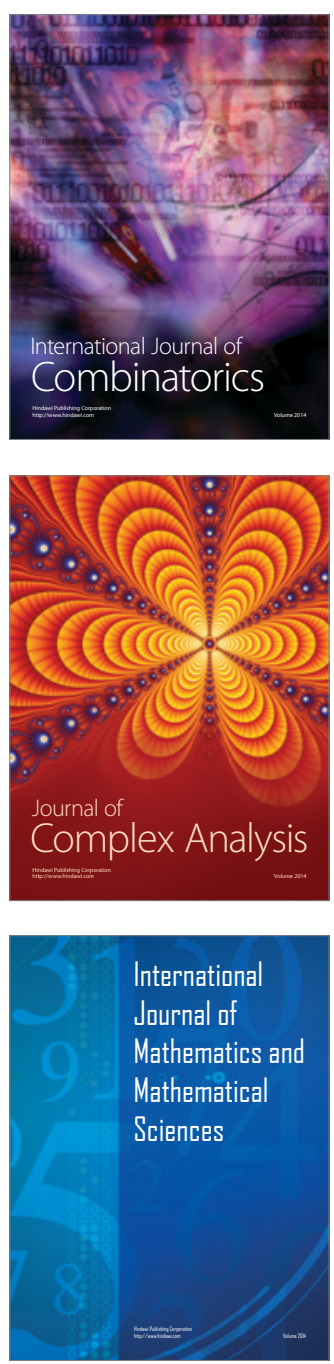
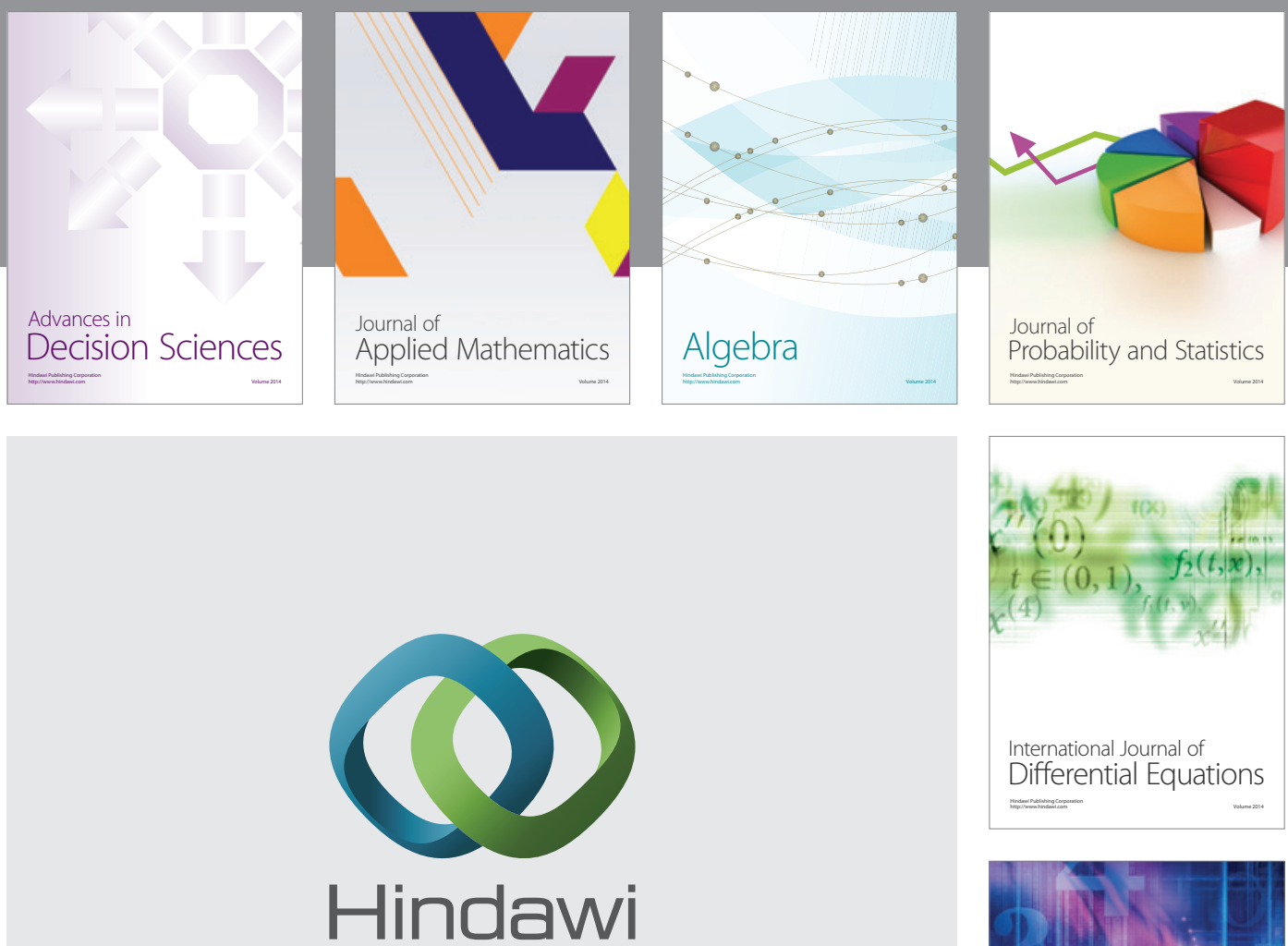

Submit your manuscripts at http://www.hindawi.com
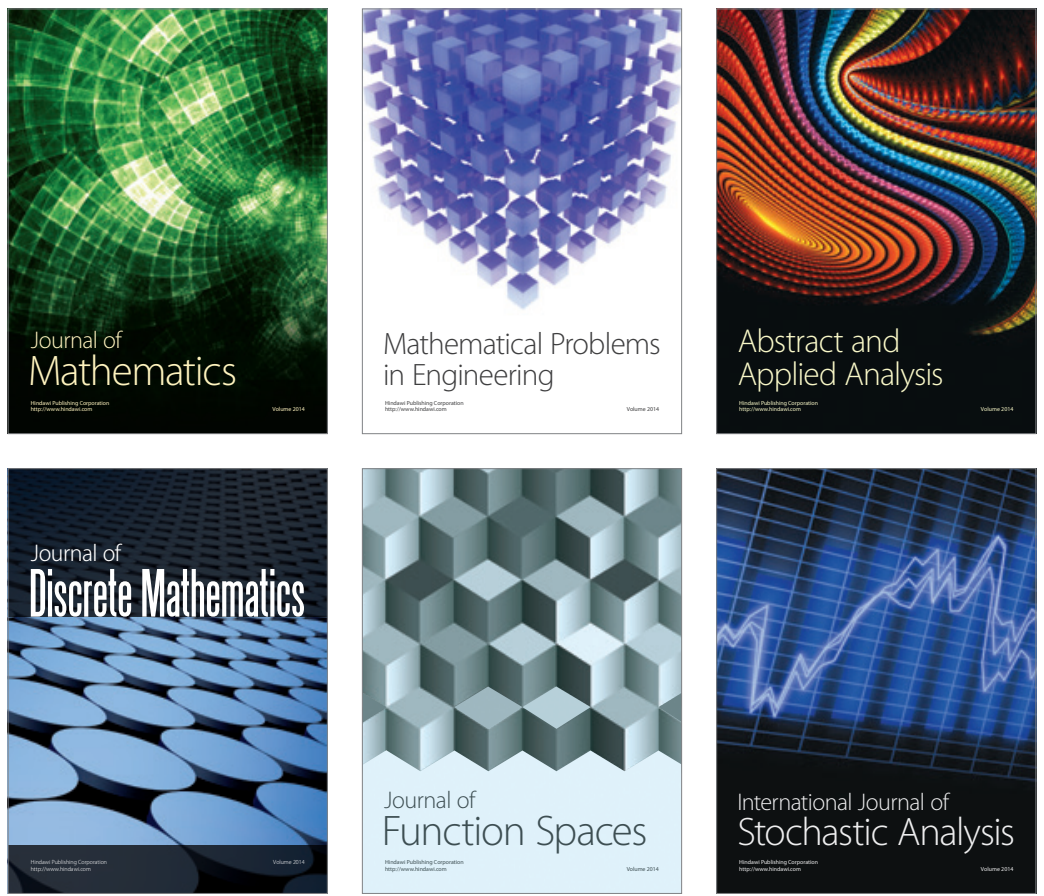

Journal of

Function Spaces

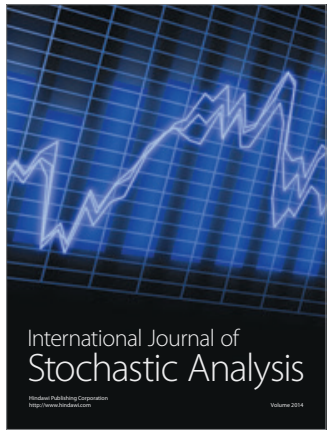

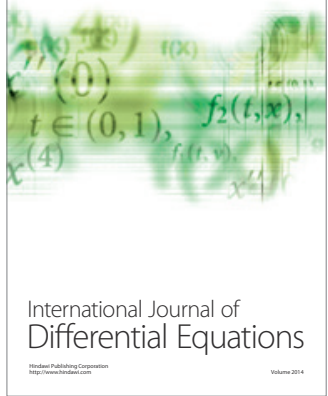
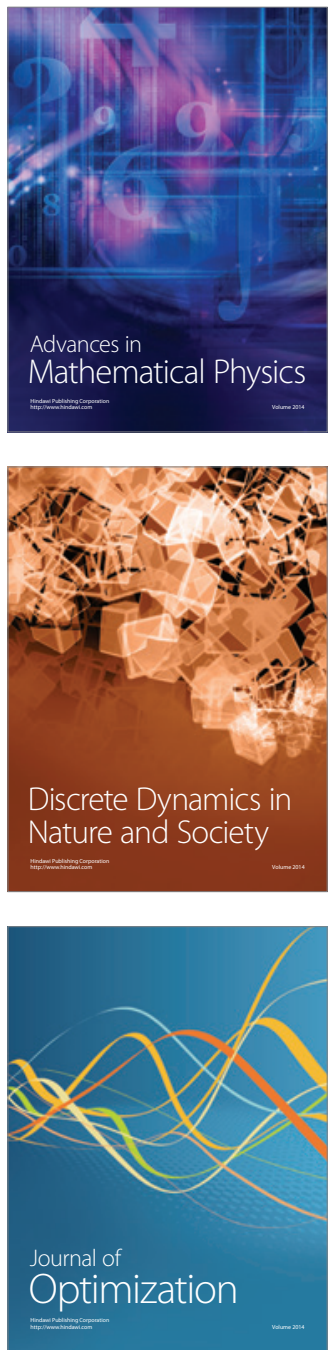